\title{
Primary Chemotherapy Resistance Malignant Mural Nodules Originated from Mucinous Borderline Ovarian Tumor: A Rare Case Report
}

Sigit Purbadi ( $\sim$ sigitpurbadi@gmail.com )

Dr. Cipto Mangunkusumo National Referral Hospital Hartono Tjahjadi

Universitas Indonesia, National Referral Hospital

Brahmana Askandar Tjokroprawiro

Dr. Soetomo National Referral Hospital

Gatot Purwoto

Dr. Cipto Mangunkusumo National Referral Hospital

Hariyono Winarto

Dr. Cipto Mangunkusumo National Referral Hospital

Gilbert Elia Sotarduga

Dr. Cipto Mangunkusumo National Referral Hospital

\section{Laurensia Scovani}

Dr. Cipto Mangunkusumo National Referral Hospital

\section{Case Report}

Keywords: Mural nodule, Ovarian Cancer, Malignant, Chemotherapy Resistance

Posted Date: February 22nd, 2022

DOI: https://doi.org/10.21203/rs.3.rs-1374843/v1

License: (c) (1) This work is licensed under a Creative Commons Attribution 4.0 International License. Read Full License 


\section{Abstract}

\section{Background}

The borderline ovarian mucinous cystic tumor may appear with mural nodules. However, its entity may remain rare. Malignant mural nodules mainly occur in old patients and have poor clinical outcomes. Accurate diagnosis of mural nodules is vital to choose the proper treatment, although it is complicated to manage.

\section{Case Presentation}

A 24-years-old nulliparous woman was examined due to abdominal pain and enlargement. She had been performed salpingo-oophorectomy due to ovarian tumor suspicion in the previous hospital. We did the surgical staging and found severe adhesion, but no cancer cell was found. During the follow-up, the symptoms got worsened. The CT-Scan results said massive ascites, CA 125 levels raise to $174 \mathrm{U} / \mathrm{mL}$, and CA 19-9 $920 \mathrm{U} / \mathrm{mL}$. Exploratory laparotomy was performed, histology examination concluded malignant mural nodules mixed type on an ovarian borderline mucinous tumor. Carboplatin 750mg, paclitaxel $300 \mathrm{mg}$, and bevacizumab $800 \mathrm{mg}$ was prescribed. In the beginning, the response was great, CA 125 and CA 19-9 levels decreased. But after four cycles, the CA 19-9 levels raise significantly. On the other hand, the CA 125 remains normal. We concluded that it was a chemotherapy resistance - malignant mural nodules and new chemotherapy regiment was prescribed but also resistance. Currently, the patient refused to continue the treatment.

\section{Conclusion}

Although it is rare, malignant mural nodules should be considered when we find a mucinous borderline ovarian tumor at a younger age. The angiosarcoma-type mural nodule is very difficult to manage due to the high probability of chemotherapy resistance.

\section{Background}

The ovarian mucinous cystic tumor may appear with mural nodules. It predominantly occurs in borderline and adenocarcinomas tumors. However, its entity may remain rare. $(1,2)$ Wide variety of mural nodules have been described as benign and malignant. Benign mural nodules are described as sarcoma-like, carcinoma-like, and rarely benign smooth muscle nodules. On the contrary, malignant mural nodules are categorized as anaplastic carcinoma, clear cell carcinoma, neuroendocrine carcinoma, giant cell carcinoma, carcinosarcoma, and sarcoma.(3) Patients with benign mural nodules (especially sarcomalike) tend to occur at a young age but should be treated with caution because of their problematic morphology, while primarily malignant occurs in the older patient and have a poor clinical outcome.(4)

It is vital to diagnose mural nodules in ovarian tumors accurately and distinguish between benign or malignant since the treatment and prognosis of each case were very different. Lack of understanding of 
clinicopathological features often leads to misdiagnosis and unreasonable treatment. In this report, we would like to show malignant mural nodules in young women and the importance of thinking of mural nodules as diagnosis or differential diagnosis when we face borderline ovarian tumor with some malignant areas continued with careful histochemical examination.

\section{Case Report}

A 24-years-old nulliparous woman was searching examination due to enlarged abdominal mass. She had no idea whether she was pregnant or not. She complained of having severe abdominal pain two weeks before admission to the previous hospital. In November 2020, the patient got a right salphingooophorectomy due to ovarian tumor suspicion. Histopathology results showed choriocarcinoma nongestational on the seromucous borderline ovarian tumor with negative peritoneal cytology result.

She got referred to us for a second opinion one month later due to a progressive ovarian cancer diagnosis. The previous doctor planned to take out all of the genital tract based on the CT-Scan that showed a mass at the right on the left stump of the ovary. Our discussion with the patient lead us to decide on conservative surgical staging, which was performed a week later. While performing the procedure, we found severe adhesion between the rectosigmoid uterus and left pelvis sidewall which is shown by the CT-Scan as recurrence mass on the left ovarian stump. The histopathology results showed multiple endometriosis nodules and a small endometriosis cyst at the right ovary and the cytology with peritoneal biopsy on pelvic and para-aortic lymph node not detected any cancer cell. Because we did not find any cancer on the surgical staging, we concluded this patient diagnosis was stage 1A ovarian cancer, and further chemotherapy was not needed. We also gave adjuvant treatment for her endometriosis. Rectal sonography follow-up every two months was prescribed.

In March 2021, the patient felt abdominal enlargement and bloating. We did the abdominal and rectal sonography, massive ascites and a small right ovarian cyst were found. Abdominal CT-Scan did not find any tumor or peritoneal carcinomatosis, but massive ascites were found. Because of these imaging findings, we suggested doing tumor marker examination, CA 125 was $174 \mathrm{U} / \mathrm{mL}$, and CA 19-9 was 920 $\mathrm{U} / \mathrm{mL}$. From our findings, we decided to do an exploratory laparotomy. Six liters of ascites fluid were evacuated, uterus was within normal limit, $3 \mathrm{~cm}$ right ovarian cyst and multiple peritoneal nodules on the peritoneum, intestine, and mesointestine were found as shown in Figure 1. We also found a small nodule in the omentum at the liver flexure line. We decided to do cystectomy and took some nodules to evaluate the histopathology. We put the active drainage to evaluate the ascites production. Paraffin block histopathology review was immediately being checked due to the peritoneal carcinomatosis. Peritoneal implant histopathology results said poorly differentiated adenocarcinoma and the immunochemistry showed different staining patterns between anaplastic and angiosarcoma (positive on CD31, P53, WT1, CD68, EMA, CAM5.2). The result for the ovarian cyst was benign. The histopathology review from primary surgery was malignant mural nodule mixed type with anaplastic and angiosarcoma type on a mucinous borderline ovarian tumor. The immunohistochemistry examination confirmed the malignant mural nodule. 
The postoperative evaluation found that the ascites production was 3 liters a day, so we treated this patient with chemotherapy on day seven. We prescribed carboplatin $750 \mathrm{mg}$, paclitaxel $300 \mathrm{mg}$, and bevacizumab (Avastin $\rightarrow$ ) $800 \mathrm{mg}$ for the chemotherapy treatment. Chemotherapy follow-up found significant yet immediately reduced of the ascites, and not produce any more after seven days, CA 125 levels remain normal $(20.9 \mathrm{U} / \mathrm{mL}), \mathrm{CA} 19-9$ level decreased until $256 \mathrm{U} / \mathrm{mL}$. PET CT-Scan found small peritoneal carcinomatosis that did not uptake fluorodeoxyglucose (FDG) / non-metabolic residual mass in the omentum, mesentery, and abdominopelvic peritoneal. After three cycles of chemotherapy, we did not find any mass or ascites was visualized on ultrasound, and serial CA19-9 evaluation on the third and fourth chemotherapy was $115,63 \mathrm{U} / \mathrm{mL}$ and $106 \mathrm{U} / \mathrm{mL}$.

After five cycles of chemotherapy, the CA $19-9$ levels increased to $327 \mathrm{U} / \mathrm{mL}$, so we decided to re-evaluate the CA 19-9 levels and prescribe PET CT-Scan evaluation. Within five days, the CA 19-9 re-examination levels showed $1054 \mathrm{u} / \mathrm{mL}$, and PET CT-Scan evaluation showed progressive disease. New multiple nodule liver metastases were found. From this evidence, we concluded that this is a primary platinum resistance tumor, and we decided to prescribe liposomal doxorubicin (Caelyx $\rightarrow$ ) 60mg, oxaliplatin (Rexta ) 150mg, and bevacizumab (Avastin ) 800mg for the chemotherapy. Response rate after one-cycle chemotherapy was awful, and the final tumor marker evaluation shows CA 19-9 2854 U/mL and CA 125 $16.40 \mathrm{U} / \mathrm{mL}$. We concluded that this is a primary platinum and second-line resistance malignant mural nodule. Currently, the patient's vital sign was normal, and no significant symptoms appear in the moment, so the patient decided to postpone all the treatment or therapy without any explanation. Due to these matters, we did counseling to the patient regarding symptomatic palliative treatment.

Table 1

CA 125 and CA 19-9 serial evaluation results

\begin{tabular}{|lll|}
\hline Date & CA 125 (U/mL) & CA 19-9 (U/mL) \\
\hline May 5th, 2021 & 174 & 920 \\
\hline June 30th, 2021 & 20.9 & 256 \\
\hline July 21st, 2021 & & 115.63 \\
\hline August 9th, 2021 & 106 \\
\hline September 1st, 2021 & 327 \\
\hline September 6th, 2021 & & 1054 \\
\hline October 6th, 2021 & 16.40 & 2854.34 \\
\hline
\end{tabular}

\section{Discussion}


Ranked as the third most common gynecologic cancer after cervical and uterine cancer, ovarian cancer has the worst prognosis and the highest mortality rate(1). Solid masses or nodules that occur in the background of ovarian neoplasms are called ovarian mural nodules, which the majority of them are seen in mucinous ovarian tumors(2). Mural nodules that occur in an ovarian tumor are considered a rare entity in a mucinous ovarian tumor, and it predominantly occurs in borderline and adenocarcinoma tumors. The literature stated there were less than 40 cases that had been reported $(3,5,6)$. Mural nodule could be benign or malignant where it is divided into several types such as sarcoma-like, carcinoma-like, or rarely benign smooth muscle mural nodules (SLMN), which are benign, and the malignant types are anaplastic carcinomatous nodules, clear cell carcinoma, giant cell carcinoma, and sarcomatous nodules. The major benign form of mural nodules is SLMN, while the most common type of mural nodules reported in malignant cases is anaplastic carcinomatous type $(4,7)$. Because of its rare occurring, mural nodules are easily missed, so it is vital to consider the possibility of its occurrence from the beginning, other than differentiating what type of the nodules are.

In our case, we presented a young woman diagnosed with mixed, anaplastic-angiosarcoma, type mural nodule. This case is not common because malignant mural nodules tend to occur in older patients with poor prognoses(7). We did not have any suspicion of a mural nodule in the first place due to the first and second operative pathological results. Also, the restaging on the second operative procedure results showed stage $1 \mathrm{~A}$ ovarian cancer. There are still limited cases of mural nodules reported in the literature. After the patient was referred to our hospital, we treated this patient with conservative management due to her young age. We did the incomplete surgical staging. Two months follow-up showed no evidence of both anatomical and tumor marker abnormality.

Our present case could be compared with a case report by Suzuki et al. that showed a malignant mural nodule in mucinous ovarian carcinoma. Mural nodule in teenage and adolescent age is unlikely, but there is still a possibility to occur in this age range(8). We suspected a case of mural nodule after the third procedure, and the patient already complained of abdominal distention resulting in massive ascites and high levels of CA 125 and CA 19-9, a biomarker for the mucinous tumor that was found in the nodules. Clinically, we concluded that it was a progressive disease. Exploratory laparotomy supports the evidence of progressive disease with massive ascites, multiple peritoneal carcinomatosis, and omental nodule. Also, strengthened by paraffin block histopathology results using hematoxylin-eosin and immunohistochemistry because most diagnostic imaging is relatively non-specific.(9)

Angiosarcoma of the ovary is rare and represents an aggressive type of malignant ovarian neoplasm, it is one of the sarcoma types which spread hematogenous, and is also less common in borderline mucinous tumors. (10) The characteristic of this kind of tumor is also resistance to chemotherapy and radiotherapy. The diagnosis of this case may be challenging due to its unspecific clinical, laboratory, or imaging features. This is when histopathology and immunohistochemistry play a vital role in definite diagnosis. Angiosarcomas typically express endothelial markers, including Factor-VIII-related antigen (Factor-VIIIRA), CD31, CD34, and vascular endothelial growth factor (VGEF). Among these markers, CD31 is the most common one with high sensitivity and specificity, therefore, considered as the gold standard in the 
immunohistochemical examination for angiosarcoma.(9) Anaplastic express CAM 5.2, AE1/AE3. AE1/AE3 expression is only positive in the anaplastic tumor. Therefore, this gene expression can be used to discriminate between sarcomatous tumors, which usually expresses positivity on Vimetin and CAM5.2.

This case is considered unique because we did not find any cancerous cell on the stump ovarian tumor on the first exploration laparotomy surgical staging. Still, we must think about the possibility of a hidden tumor due to massive inflammation that showed from wide fibrotic tissue, and the elevated of IL-6 levels raises into $300 \mathrm{pg} / \mathrm{mL}$. Tumor marker, CA 125 and CA 19-9, with PET-Scan evaluation, may be helpful to determine the chemotherapy response. At the beginning of this case, we found that the response of chemotherapy was great, proven by a significant decrease of CA 125 and CA 19-9 levels. The PET-Scan evaluation said non-metabolic mass. It may be indicated that the tumor masses may be dispersed. As a result, the tumor metabolism was not detected. This suspicion was supported by the third and fourth chemotherapy CA 19-9 evaluation, which decreased very slightly. This raises the idea that the chemotherapy response was less significant and prospectively. The fifth evaluation shows an increase of 19-9 levels. We concluded that this tumor was a platinum resistance one, and we changed the chemotherapy to liposomal doxorubicin, oxaliplatin, and bevacizumab. The chemotherapy response after only one cycle was awful. The CA 19-9 levels raised significantly, concluded that this was a progressiveplatinum resistance malignant mural nodule which is extremely rare.

Theoretically, malignant mural nodules may display a sarcomatoid, rhabdoid, or pleomorphic appearance that reveals their epithelial derivation(12). A malignant mural nodule that originated from ovarian carcinoma usually showed a poor prognosis, a $50 \%$ mortality rate. Still, another study stated there is no relation in histological pattern between anaplastic nodules and prognosis of the disease $(3,13,14)$. Like previous cases, this case shows the progressivity and the poor prognosis of the malignant mural nodule, an anaplastic - angiosarcoma mixed type in our case. The anaplastic and angiosarcoma type of mural nodule ovarian tumor is characterized as high in aggressivity and less sensitive to chemotherapy.

This kind of mural nodule is complicated to manage and easily infiltrate with the high rate of local recurrence, chemotherapy resistance possibility, and metastasis. Due to this matter, even though it is rare, if we found a nodule in the borderline ovarian tumor, we should the possibility of a mural nodule and evaluate its type.

\section{Conclusion}

Mural nodules should also be considered when we find mucinous borderline ovarian tumors at a younger age. Although it is rare, the possibility of its occurrence still exists. Earlier diagnosis could lead to a suitable therapy regimen and a better outcome. Nevertheless, the angiosarcoma type mural nodule is very difficult to manage due to the high probability of chemotherapy resistance.

\section{Declarations}




\section{Ethical Approval}

This study was reviewed and approved by the Institutional Review Board and Ethical Committee, Dr. Cipto Mangunkusumo, a national reference, and teaching hospital. Patient medical records were maintained under applicable medical ethical standards.

\section{Patient Consent}

Written informed consent was obtained from the patient for publication of this case report and accompanying images. A copy of the written consent is available for review by the Editor-in-Chief of this journal on request.

\section{Availability of data and materials}

Not applicable

\section{Competing of Interest}

The authors declare that we have no financial or competing interests that may have inappropriately influenced us in writing this article.

\section{Sources of Funding}

This research did not receive any specific grant from funding agencies in the public, commercial, or notfor-profit sectors.

\section{Authors' Contributions}

SP: conceptualization, investigation, methodology, resources, supervision. GES: writing-original draft preparation, investigation, visualization, writing-review and editing. HT: investigation, supervision, resources. BAT: investigation, resources. GP, HW, LS: supervision, data curation, editing. All authors read and approved the final manuscript.

\section{Acknowledgements}

Not applicable

\section{References}

1. Momenimovahed Z, Tiznobaik A, Taheri S, Salehiniya H. Ovarian cancer in the world: epidemiology and risk factors. International Journal of Women's Health. 2019 Apr; Volume 11.

2. Lakshmi Haridas K, Menon A, Deepthi B. Borderline ovarian mucinous tumor with anaplastic carcinomatous mural nodule: A case report. Gynecologic Oncology Reports. 2021 Aug;37. 
3. Desouki MM, Khabele D, Crispens MA, Fadare O. Ovarian Mucinous Tumor With Malignant Mural Nodules. International Journal of Gynecological Pathology. 2015 Jan;34(1).

4. Chapel DB, Lee EK, da Silva AFL, Teschan N, Feltmate C, Matulonis UA, et al. Mural nodules in mucinous ovarian tumors represent a morphologic spectrum of clonal neoplasms: a morphologic, immunohistochemical, and molecular analysis of 13 cases. Modern Pathology. 2021 Mar 5;34(3).

5. Zhang Y, Yuan Z, Sun K, Li P. Ultrasonic and pathological characteristics of ovarian mucinous cystic tumors with malignant mural nodules. Medicine. 2017 Nov;96(45).

6. Desouki MM, Fadare O, Kanbour A, Kanbour-Shakir A. Immunophenotype and K-RAS Mutation in Mucinous Ovarian Adenocarcinoma With Mural Nodule of High-grade Sarcoma. International Journal of Gynecological Pathology. 2014 Mar;33(2).

7. Shao Y, Liu Q, Shi H, Lu B. Ovarian mucinous tumors with mural nodules: immunohistochemical and molecular analysis of 3 cases. Diagnostic Pathology. 2020 Dec 14;15(1).

8. Suzuki K, Imada H, Igarashi A, Saitou Y, Takakura S. Ovarian Mucinous Carcinoma with Mural Carcinosarcomatous Components in a Prepubertal Girl. Journal of Pediatric and Adolescent Gynecology. 2019 Aug;32(4).

9. Cao J, Wang J, He C, Fang M. Angiosarcoma: a review of diagnosis and current treatment. American journal of cancer research. 2019;9(11).

10. Khunamornpong S, Settakorn J, Sukpan K, Pongsuvareeyakul T, Siriaunkgul S. Angiosarcoma Arising in Ovarian Mucinous Tumor: A Challenge in Intraoperative Frozen Section Diagnosis. Case Reports in Pathology. 2016;2016.

11. Okumura T, Muronosono E, Tsubuku M, Terao Y, Takeda S, Maruyama M. Anaplastic carcinoma in ovarian seromucinous cystic tumor of borderline malignancy. Journal of Ovarian Research. 2018 Dec 3;11(1).

12. Vroobel KM, Attygalle AD. Uncommon ovarian epithelial tumours. Diagnostic Histopathology. 2020 Jun;26(6).

13. Provenza C, Young RH, Prat J. Anaplastic Carcinoma in Mucinous Ovarian Tumors. American Journal of Surgical Pathology. 2008 Mar;32(3).

14. Allende DS, Drake RD, Chen L. Mural Nodules of Clear Cell Carcinoma in a Mucinous Borderline Tumor of the Ovary: A Case Report. Pathology Research International. 2010 Apr 13;2010.

\section{Figures}




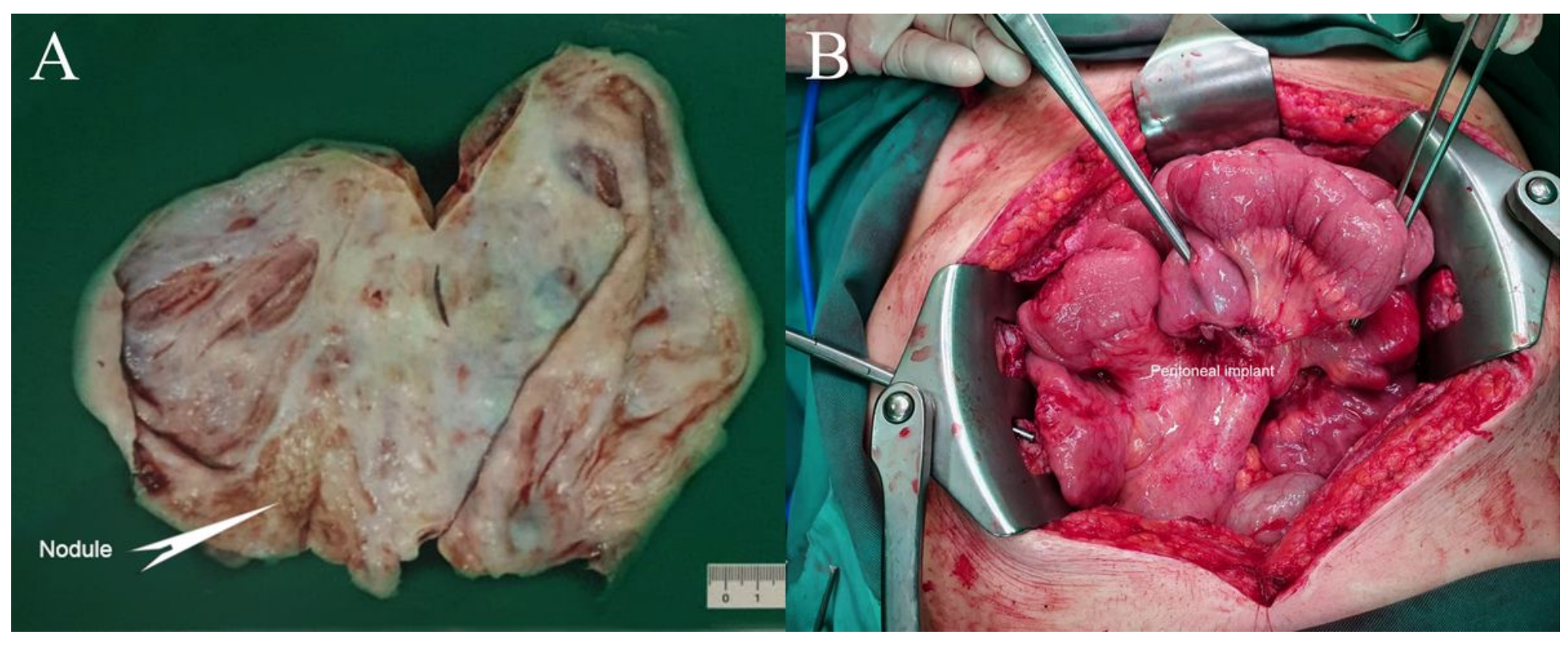

\section{Figure 1}

Macroscopic appearance; $A$. The tumor macroscopic appearance showed nodules in ovarian mass characteristically spongy to solid mass without hemorrhagic foci; $B$. Peritoneal implant was found in laparotomy procedure. 


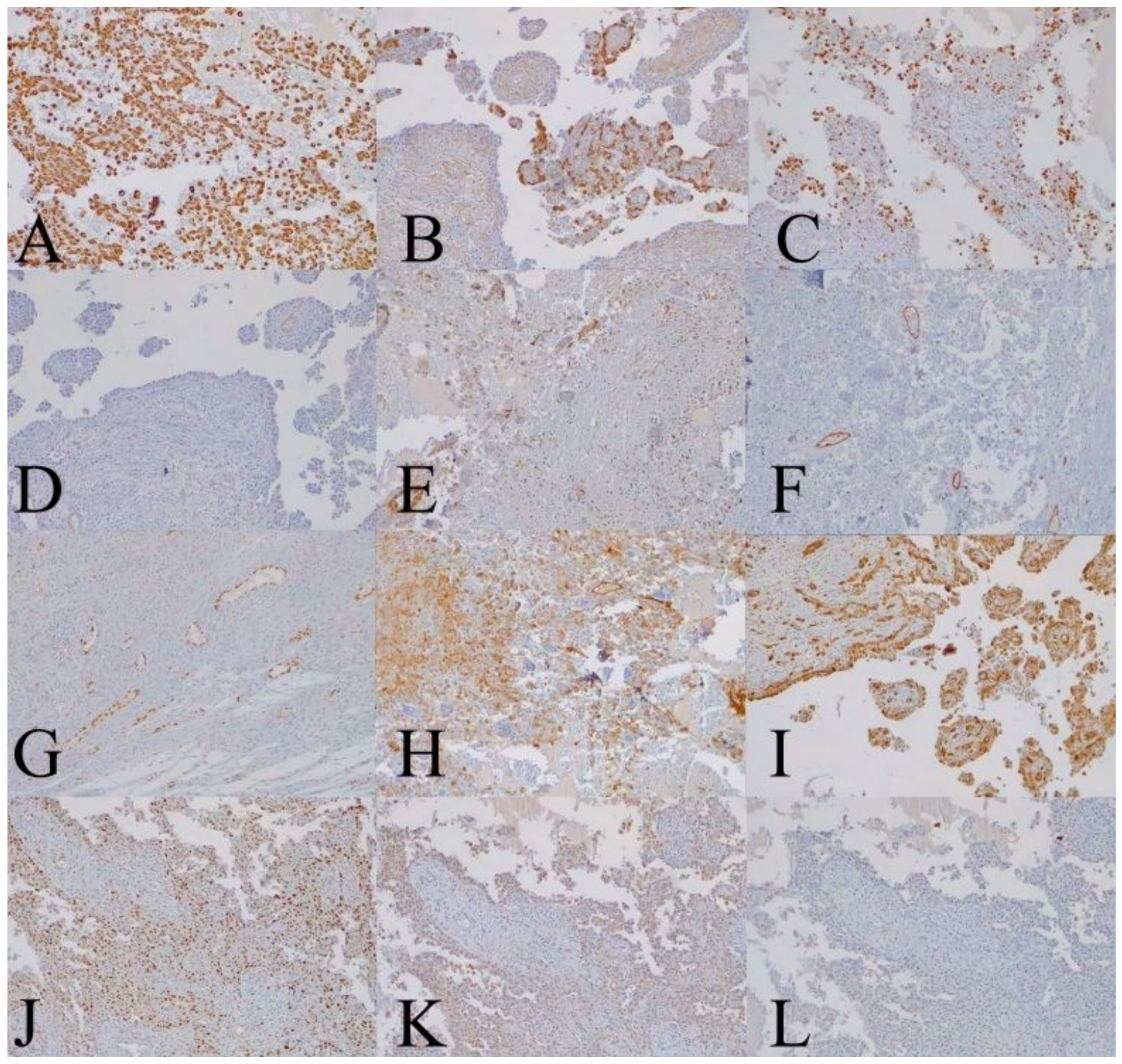

\section{Figure 2}

Immunohistochemistry Results; A. CAM 5.2: positive on mural nodule tumor mass; B. EMA: positive on mural nodule tumor mass; C. CAM 68: positive on macrophage cells; D. Desmin: negative; E. MSA: negative; F. CD 34: negative in the tumor cells, positive in the endotel; G. ERG: negative in tumor cells, positive in the endotel; $\mathrm{H}$. CD 31: focal positive in the tumor cells, positive in the endotel; I. WT 1: positive; J. p53: positive (mutation type pattern); K. Napsin A: negative / non-specific; L: p40: negative 


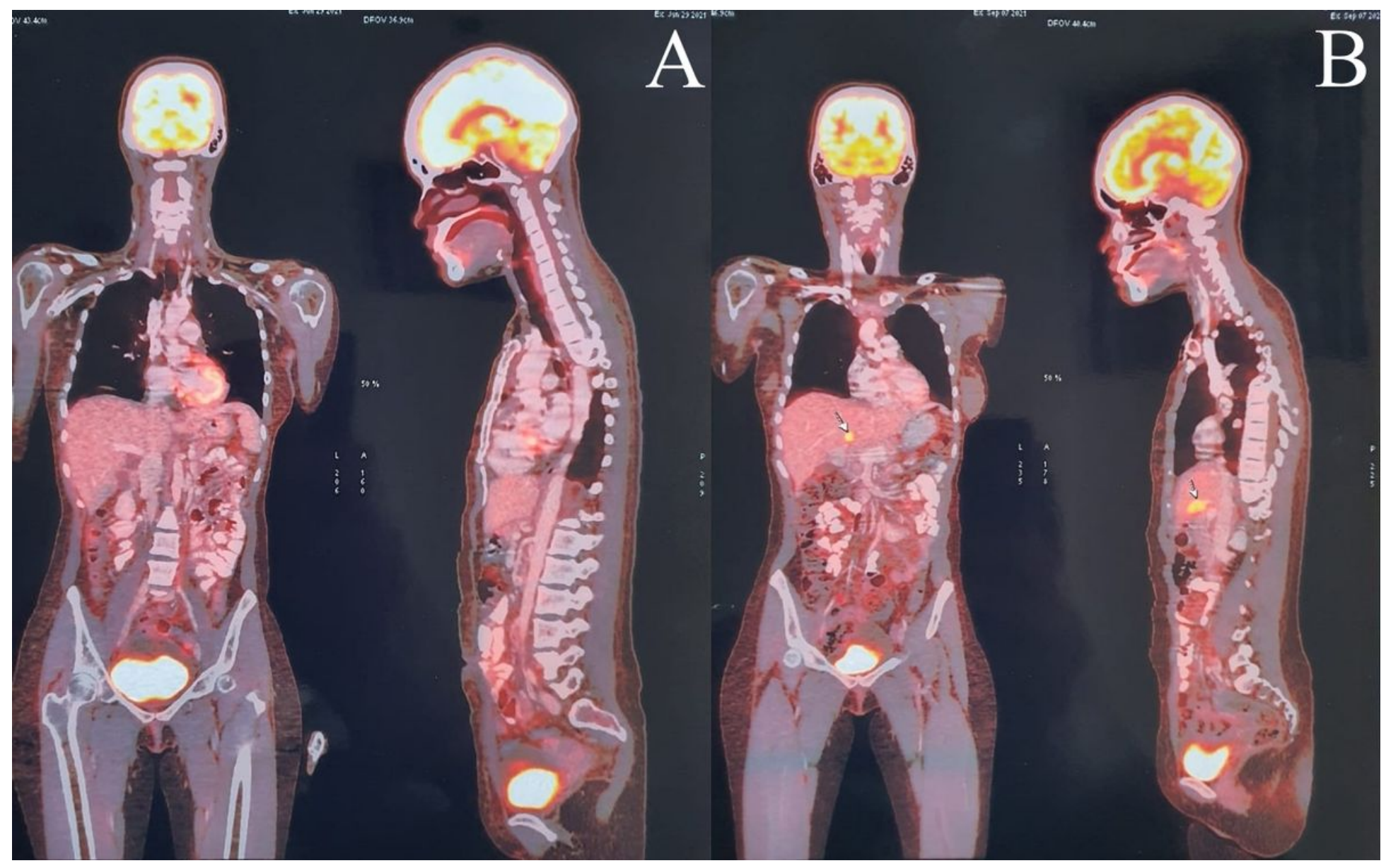

\section{Figure 3}

PET-Scan Evaluation Results. (A) No liver metastatic in the first evaluation; (B) New multiple intrahepatic, perihepatic and mesenteric metastatic nodule detected, suggested progressive disease after five cycles of chemotherapy in the three-month PET-Scan evaluation.

\section{Supplementary Files}

This is a list of supplementary files associated with this preprint. Click to download.

- Highlights.docx 